\title{
A three-step procedure for analysis of circumplex models: An example of narcissism located within the circumplex of personality metatraits
}

\author{
Rogoza, Radosław ; Cieciuch, Jan ; Strus, Włodzimierz
}

\begin{abstract}
Circumplex models are widely utilized in the field of personality and individual differences research. Although within the literature one could find many suggestions on how to analyze such models, none of them are comprehensive enough. Within the current paper we propose a three-step procedure, which will systematize and standardize the analysis of circumplex models and the localization of external variables within such circumplex models. First, we propose to verify the underlying circumplex structure through structural equation modeling. Second, we propose to test the possibility to locate external variables in the space of the empirical circumplex model through the investigation of their structural summary profiles. Finally, we propose to test the congruence between empirical locations and theoretical predictions within the circumplex structure through the Procrustes rotation. The three-step procedure is described using the example of narcissism embedded within the Circumplex of Personality Metatraits model. This paper is supplemented by pedagogical tutorials assisting other researchers in applying the three-step procedure to their own data.
\end{abstract}

DOI: https://doi.org/10.1016/j.paid.2019.109775

Posted at the Zurich Open Repository and Archive, University of Zurich

ZORA URL: https://doi.org/10.5167/uzh-182629

Journal Article

Accepted Version

Originally published at:

Rogoza, Radosław; Cieciuch, Jan; Strus, Włodzimierz (2021). A three-step procedure for analysis of circumplex models: An example of narcissism located within the circumplex of personality metatraits. Personality and Individual Differences, 169:109775.

DOI: https://doi.org/10.1016/j.paid.2019.109775 
A Three-Step Procedure for Analysis of Circumplex Models:

An Example of Narcissism located within the Circumplex of Personality Metatraits

Radosław Rogoza

Cardinal Stefan Wyszyński University in Warsaw

Jan Cieciuch

Cardinal Stefan Wyszyński University in Warsaw \& University of Zurich

Włodzimierz Strus

Cardinal Stefan Wyszyński University in Warsaw

\begin{abstract}
Author Note
Correspondence concerning this article should be addressed to Radosław Rogoza, Institute of Psychology, Cardinal Stefan Wyszyński University, Wóycickiego 1/3, 01-938 Warsaw, Poland. Email: r.rogoza@uksw.edu.pl. The data, syntaxes, and tutorials are available at: https://osf.io/r95h7.
\end{abstract}

Word count: 5280 
Graphical Abstract (for review)

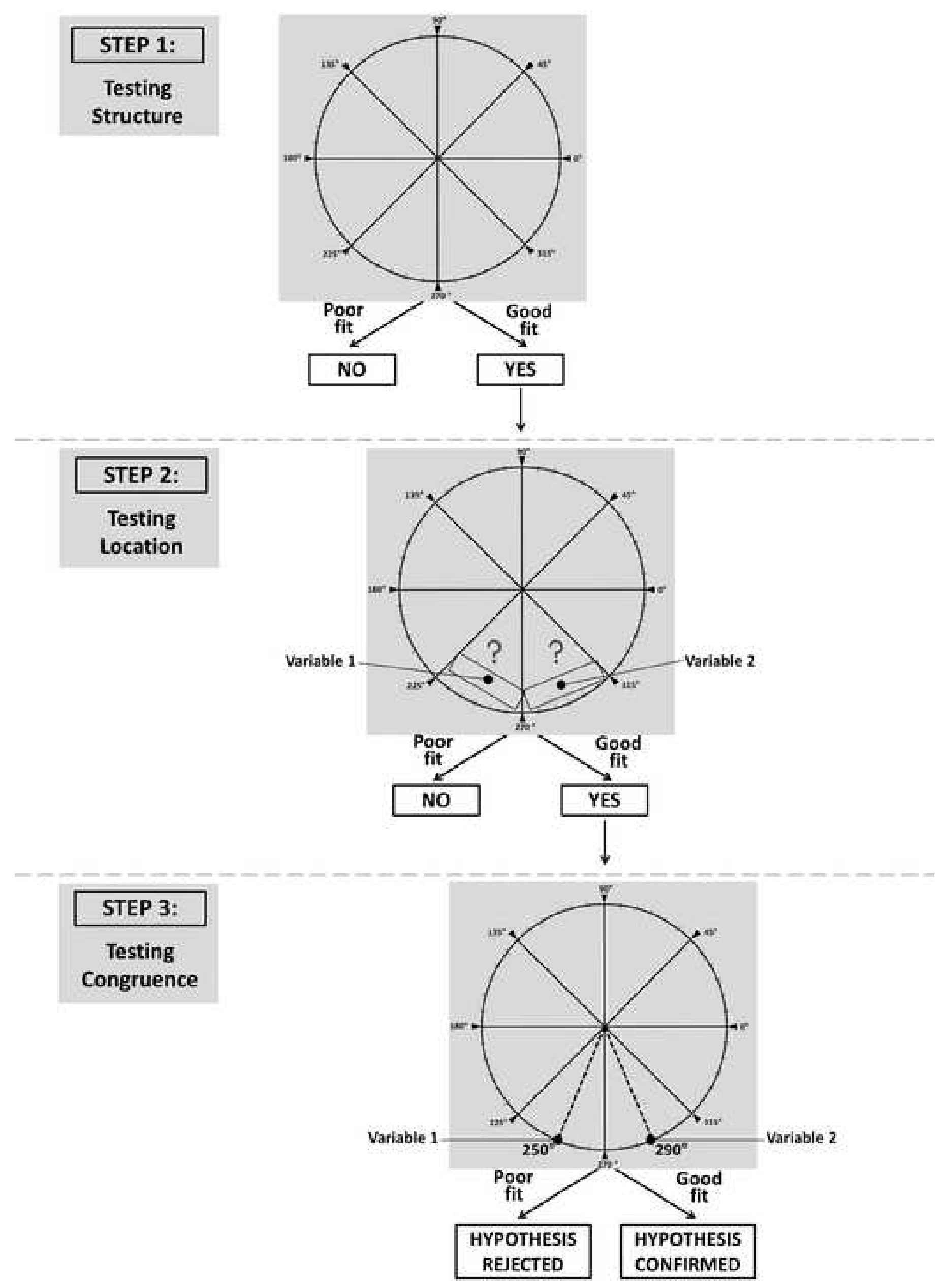

STEP 2:

Testing Location

STEP 3:

Testing Congruence

ture

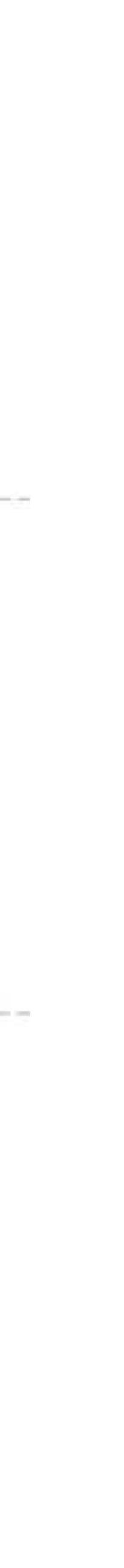




\section{Highlights}

-> A three-step procedure for analysis of circumplex models has been proposed

-> Step 1: Testing of the circumplex structure

-> Step 2: Testing the possibility to locate an external variable within circumplex

-> Step 3: Testing congruence between empirical locations and theoretical expectations 


\begin{abstract}
Circumplex models are widely utilized in the field of personality and individual differences research. Although within the literature one could find many suggestions on how to analyze such models, none of them are comprehensive enough. Within the current paper we propose a three-step procedure, which will systematize and standardize the analysis of circumplex models and the localization of external variables within such circumplex models. First, we propose to verify the underlying circumplex structure through structural equation modeling. Second, we propose to test the possibility to locate external variables in the space of the empirical circumplex model through the investigation of their structural summary profiles. Finally, we propose to test the congruence between empirical locations and theoretical predictions within the circumplex structure through the Procrustes rotation. The three-step procedure is described using the example of narcissism embedded within the Circumplex of Personality Metatraits model. This paper is supplemented by pedagogical tutorials assisting other researchers in applying the three-step procedure to their own data.
\end{abstract}

Keywords: Circumplex Models; Structural Equation Modeling; Structural Summary Method; Procrustes rotation; Narcissism 


\section{Introduction}

\subsection{Defining the Circumplex Model}

Individual differences are conceptualized in models usually composed of a set of variables. Taking into account the relations between the variables, two kinds of models can be distinguished. In the first type, relations between variables are not strictly determined, while in the second type the relations between variables are precisely described based on theoretical reasoning. In the first type of models the necessary requirement is the differentiability between variables. As an example, take the Dark Triad (Paulhus \& Williams, 2002). Narcissism, psychopathy and Machiavellianism are expected to be related to each other (because they describe a common phenomenon), but not too much (because they focus on various aspects of the described phenomenon). Such models are usually tested through the means of factor analysis. In the second type of models, two requirements matter. In addition to the differentiability between variables, precisely determined relations between variables are expected. There are several subtypes of such models. In some of them the variables are claimed to be orthogonal, thus the correlations between variables are expected to be zero. This is, for example, the case for the Big Five (McCrae \& Costa, 1997). A special case of models within this type are circular and circumplex models. In such models, there are several expectations regarding the relations between variables. Some variables are expected to be orthogonal (not correlated), some variables are expected to be positively correlated, while others are expected to be negatively correlated and some variables are expected to be more correlated than others.

The circumplex model could be defined through the terms of: 1) the possibility to locate variables differentiated in the model in the two-dimensional space with 2) equal communality (i.e., same distance between variables from the middle of the circle); and 3) equal spacing (i.e., same distance between variables within the model around circumplex; 
Gurtman, 1994; Gurtman \& Pincus, 2003). On the basis of these criteria, one could differentiate four models, depicted on Figure 1: a circular one (less restrictive Model 1), quasi-circumplex (Model 2 and 3) and circumplex (most restrictive Model 4).
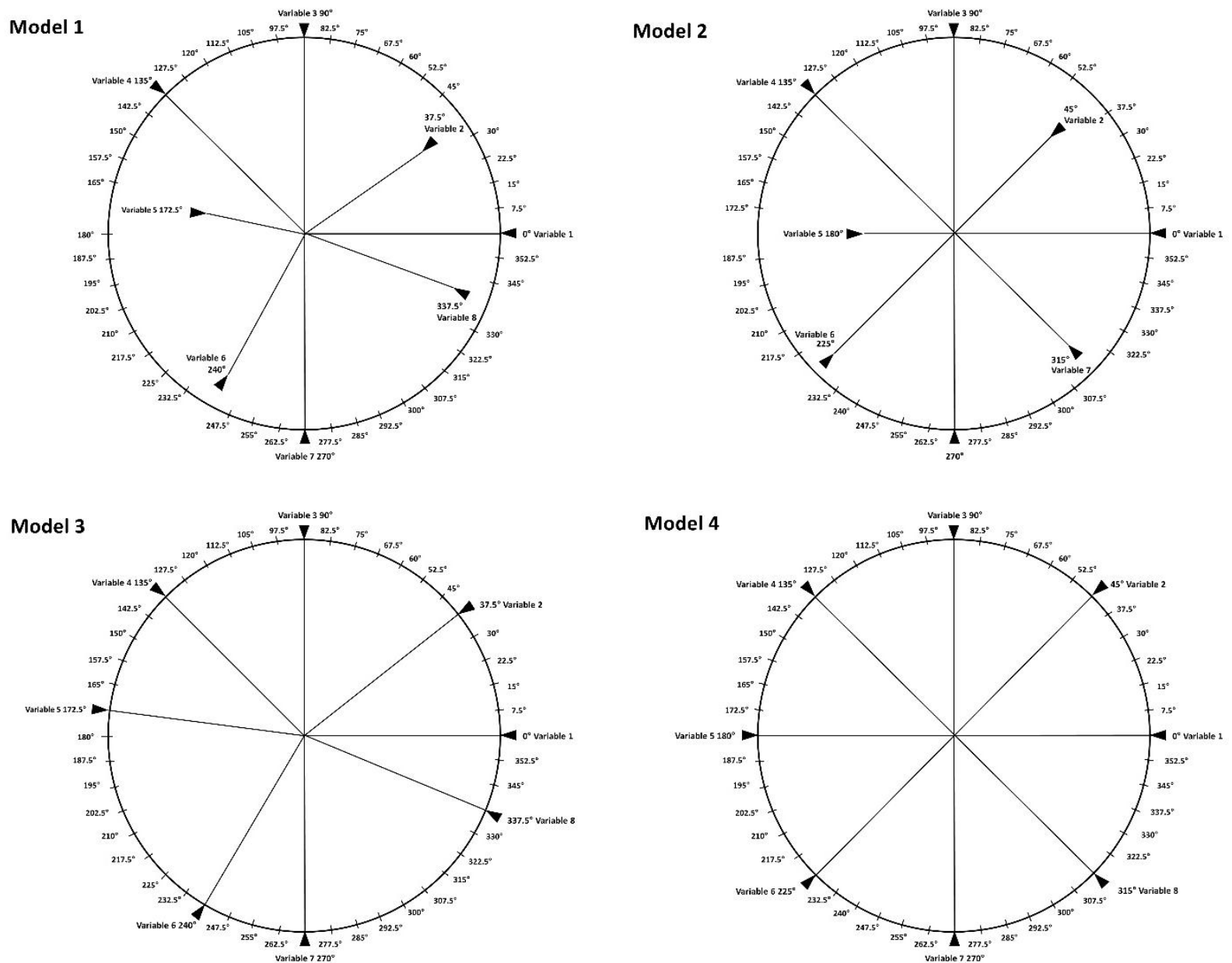

Figure 1. Graphical Representations of a Circular Model (1), Quasi-Circumplex Models (2 and 3), and Circumplex Model (4).

In Model 1 all variables are assigned in a circular order, but they are not equally spaced or have equal communality. Such models are called circular. Two models fulfil one of the requirements mentioned above but not another, that is: variables in Model 2 are located with equal spacing but unequal communalities, while variables in Model 3 are located with unequal spacing but equal communalities. These models are referred to as quasi-circumplex. 
Finally, there is Model 4, where both spacing and communalities are equal. This model is referred to as circumplex.

\subsection{Locating External Variables Within Circumplex Models}

Circumplex models allow for the testing of complex hypotheses not only about relations between circumplex variables, but also about relations with external variables. Therefore, the analyses include two parts. The first one is testing the internal structure of the model in order to confirm its circumplex nature. The second one is the analysis of relations between external variables with variables differentiated in the circumplex or, in other words, these variables could be located within the space defined by the circumplex. This part is especially interesting because locating variables allows hypotheses to be formulated regarding all circumplex variables simultaneously. For example, some of the relations between a particular variable and all variables from a circumplex model would be expected to be strong, some weak, some negative, and some positive. The circumplex model precisely predicts all of them.

Circumplex models usually describe a given area of psychological functioning in a comprehensive way. For example, the assumption of the interpersonal circumplex (Wiggins, 1979 ) is that it describes the whole range of interpersonal behaviors. The same concerns other circumplex and circular models like values (Schwartz et al., 2012), affects (Russel, 1980) or identity formation modes (Cieciuch \& Topolewska, 2017), to mention just a few. Thus, locating an external variable within the space of a circumplex enables interpretation of this variable in the context of the comprehensive description of a given psychological area of interest. A special case within circumplex models that enables such an interpretation, albeit exceptionally far reaching, is the Circumplex of Personality Metatraits (Strus, Rowiński, \& Cieciuch, 2014; Strus \& Cieciuch, 2017). 


\subsection{A Special Case of Circumplex Models - The Circumplex of Personality Metatraits}

The Circumplex of Personality Metatraits (CPM, illustrated on Figure 2) was based on the Five Factor Model of personality (McCrae \& Costa, 1997), although it offers a more parsimonious description of personality as the five-dimensional space is transformed into a two-dimensional one. Turning from the research on the Five Factor Model metatraits of Alpha/Stability and Beta/Plasticity (Cieciuch \& Strus, 2017) in a circumplex model solved controversies around the General Factor of Personality (Gamma/Integration in CPM; Revelle \& Wilt, 2013) as it is no longer hypothesized to be located at the top of the hierarchy of personality but at the same level of the circumplex structure (Strus et al., 2014). Moreover, another metatrait (labelled Delta/Restraint), as a result of high/low Plasticity versus low/high Stability, was distinguished to fully cover the conceptual space of the circumplex. Metatraits within CPM are unipolar dimensions, that is, each metatrait introduces qualitatively distinct psychological content (as in supplementary materials). Therefore, there are eight metatraits within the model: Alpha, Beta, Gamma, and Delta, with a positive pole labeled as Plus and a negative pole as Minus. All of the metatraits within CPM have equal communalities and are equally spaced by $45^{\circ}$. 


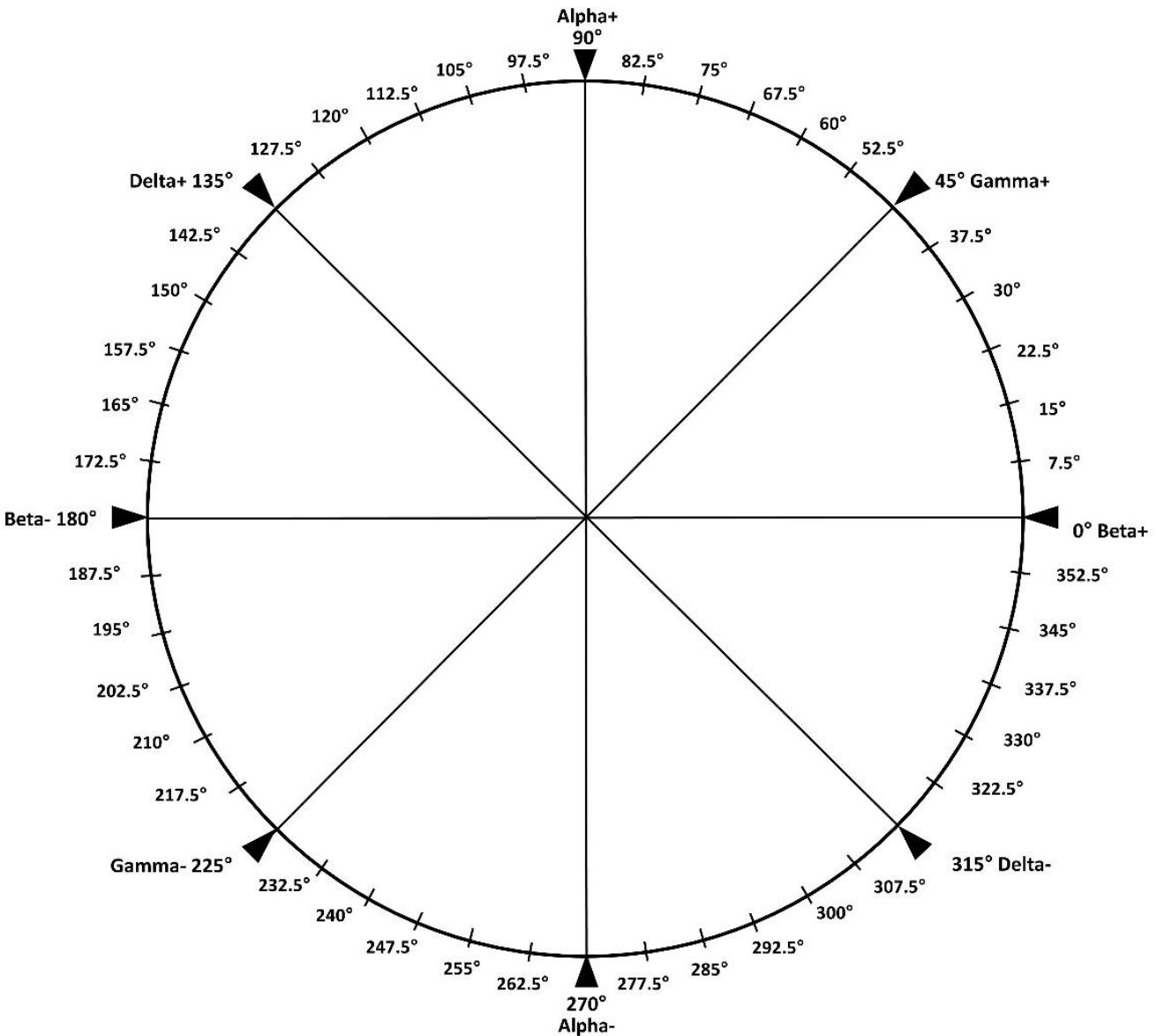

Figure 2. Graphical Representation of the Circumplex of Personality Metatraits.

CPM has two advantages over the other models (Strus \& Cieciuch, 2017): 1) it provides the possibility to grasp the comprehensive personality underpinnings for various psychological constructs in terms of the broadest personality dimensions and 2) it creates the opportunity to capture, clarify, and systematize the relationships between a very broad range of various psychological constructs. These advantages of the CPM have already been utilized within the literature to clarify relations between a range of constructs, including personality disorders (Zawadzki, 2017), and more specifically, narcissism (Rogoza, Cieciuch, Strus, \& Baran, 2019), which will serve as an example for the tutorial purposes of the current paper. 


\subsection{Structure of Narcissistic Personality Within the Circumplex of Personality}

\section{Metatraits}

Within the literature, it is widely agreed that narcissism has a three-factorial structure composed of: grandiosity (i.e., exaggerated self-views), antagonism (i.e., superiority and lack of respect towards others) and vulnerability (i.e., hypersensitivity and reactiveness; Wright \& Edershile, 2018). One of the theoretical models, that is, the Narcissism Spectrum Model (NSM; Krizan \& Herlache, 2018), describes the structure of narcissistic personality in terms of angles. Grandiosity and vulnerability are both located at an approximately angle of $45^{\circ}$ from antagonism (labeled as self-importance in NSM), which is located at the core of the narcissistic personality. Such a location implies that grandiosity and vulnerability should be unrelated to one another while antagonism should be positively related to both. This is in high congruence to the reports found within the literature (Rogoza, Żemojtel-Piotrowska, Kwiatkowska, \& Kwiatkowska, 2018). Rogoza and colleagues (2019) investigated whether NSM could be located within the conceptual space of the CPM. Indeed, using a vast array of narcissism measures, the assumed location (illustrated on Figure 3) was confirmed. 


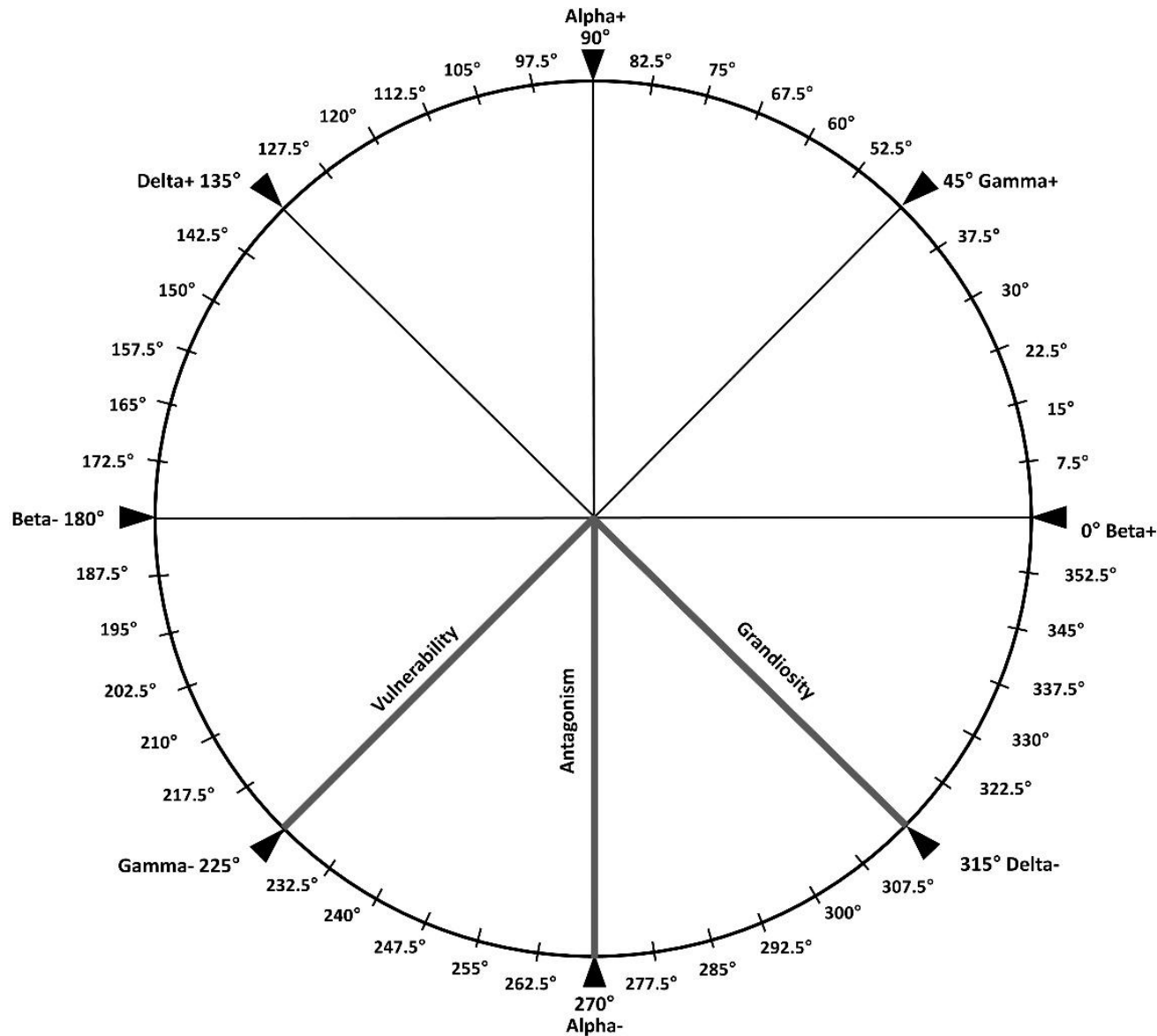

Figure 3. Location of the Narcissism Spectrum Model Within the Circumplex of Personality Metatraits.

The NSM is located from Gamma-Minus (vulnerability), through Alpha-Minus (antagonism) to Delta-Minus (grandiosity). These locations correspond to both theoretical expectations and previous empirical results, providing the first truly comprehensive description of the underlying general personality underpinnings of narcissistic personality (Rogoza et al., 2019). Most of the existing narcissistic personality measures were examined within the study, except for the Five Factor Narcissism Inventory (Miller et al., 2016), the dimensions of which, namely agentic extraversion (i.e., grandiosity), self-centred antagonism, and narcissistic neuroticism (i.e., vulnerability; Crowe, Lynam, Campbell, \& Miller, 2019), are hypothesized to correspond to the NSM dimensions (Wright \& Edershile, 2018).

Therefore, the empirical added value of this paper is to test the location of the FFNI subscales 
within the framework of the CPM. We are doing this as an example of the three-step procedure to analyze the circumplex models we propose.

\section{A Three Step Procedure for the Analysis of Circumplex Models}

We propose a three-step procedure to analyze the circumplex models. The first is to verify whether the analyzed model is circumplex or not. The second one is to locate external variables within the circumplex. The third one is to assess the extent of which empirical locations are in congruence to the theoretical predictions within the circumplex structure. The details of each step are described below.

\subsection{Step 1. Testing of the Circumplex Structure - Structural Equation Modeling}

Verification of the circumplex structure is based on the circular stochastic process model with a Fourier series correlation function ${ }^{1}$ proposed by Browne (1992). It is a nonstandard structural equation model (SEM), in which the underlying structure of intercorrelations is represented as an ordering along the circumference (Grassi, Luccio, \& Di Blas, 2010). Four models can be tested: 1) Circular: unequal spacing and unequal communality model; 2) Quasi-circumplex with equal spacing but unequal communality; 3) Quasi-circumplex with unequal spacing and equal communality; and 4) Circumplex: equal spacing and equal communality. Achieving good model fit for a more constrained model implied no need to test less restricted models.

\subsubsection{Criteria of Evaluation}

To evaluate model fit, standard fit indices used in SEM are used, that is: $\chi^{2}$ test, Comparative Fit Index (CFI) > .90; Goodness of Fit Index (GFI) > .90; Adjusted Goodness of

\footnotetext{
${ }^{1}$ The model allows to specify additional free parameters (reflecting the number of betas in the Fourier correlation function), permitting a larger number of possible correlation functions to be fit to the data (Browne, 1992). More precisely, one might analyze a model having from one to four betas, adding each frees up one model parameter. We recommend running each specification, choosing the one which had converged and had best fit.
} 
Fit Index $($ AGFI $)>.85$; Root Mean Square Error of Approximation $($ RMSEA) $<.08$ (Byrne, 1994; Schermelleh-Engel, Moosbrugger, \& Müller, 2003). However, it is worth noting that the RMSEA may not be best suited to evaluate circumplex models. It becomes biased in the case of high correlations between proximal variables, as found in circumplex models, and should be interpreted with caution (Browne, MacCallum, Kim, Andersen, \& Glasser, 2002; Saris, Satorra, \& van de Veld, 2009). We recommend interpretation of RMSEA with greater caution, similar to Gurtman and Pincus (2003) who did not interpret RMSEA $<.13$ as an indicator of poor fit, given that other fit indices suggested good model fit.

\subsection{Step 2. Testing the Possibility to Locate an External Variable Within Empirical Circumplex - Structural Summary Method}

The circumplex model precisely predicts relations between an external variable with all circumplex variables, which usually assume a sinusoidal character. To describe these relations, Gurtmann (1994) introduced the structural summary method (SSM). As the correlation pattern between an external variable to circumplex variables is sinusoidal, it could be modeled against a cosine function (Gurtman \& Pincus, 2003). The SSM estimates are: 1) model fit, informing how well the observed sinusoidal curve fits the cosine function; 2) elevation, which is the average correlation between an external variable and all circumplex variables. It represents the size of the general factor, sometimes observed in circumplex models (Tracey, 2000); 3) amplitude (i.e., vector length), which is the distance between an external variable's mean and peak correlation to the circumplex variables. It represents the distinctiveness of the profile, that is, how much it is peaked (i.e., is it more specifically related to one of the circumplex variables?) versus flat (i.e., is it similarly related to all circumplex variables?; Zimmerman \& Wright, 2017); and 4) angular displacement is the angle at which the profile reaches its highest point. It represents the empirical location of the variable within the empirical circumplex found in the data. In addition to that, SSM also computes the 
estimates of distance from $\mathrm{X}$ - and $\mathrm{Y}$-axes, corresponding to the basic dimensions of a given circumplex. All of the estimates from SSM are provided with their lower and upper confidence intervals.

\subsubsection{Criteria of Evaluation}

First, one needs to assess the goodness of fit of the sinusoidal curve to the cosine

function $\left(R^{2}\right)$. If its values are below .70 , the remaining coefficients should not be interpreted and the locating of external variables aborted, while values above .80 suggest good fit (Wright, Pincus, Conroy, \& Hilsenroth, 2009). Estimates of amplitude (i.e., is the profile distinct?) and elevation (i.e., is there a general factor?) above .15 are notable, whereas angular displacement assumes values from 0 to 360 , reflecting empirical location on the circumplex found in the data.

\subsection{Step 3. Testing Congruence Between Empirical Locations and Theoretical Expectations Within Circumplex Structure - Procrustes Rotation}

The results of SSM provides information on how an external variable is located in an empirical circumplex, that is, in the circumplex as it appears in the analyzed data. To answer whether these empirical locations meet the theoretical expectations, we propose using an orthogonal Procrustes rotation (Schönemann, 1966). Its goal is to compare the degree to which the empirical matrix is congruent with theoretical one. The theoretical matrix is derived from the transformation of the theoretical angles using sine and cosine functions. As a result, one obtains a theoretical matrix with two factors. The first factor corresponds to the estimates of sines, and the second factor corresponds to the estimates of cosines. The empirical matrix is derived from the transformation of the empirical angles (i.e., angular displacement in SSM), which also uses trigonometric functions and is saved in the form of factor loadings. 
As these matrices are expressed in the same formal language, it is possible to compare their congruence through aligning the empirical matrix to the theoretical by orthogonal rotation of the first one against the second. This is done without changing the relative location of factors, which means that the empirical results remain unchanged (Terracciano, McCrae, Hagemann, \& Costa, 2003). In other words, this rotation allows the precise testing of whether empirical locations are congruent to the expectations. A simplified illustration of how Procrustes rotation works is presented in Figure 4.
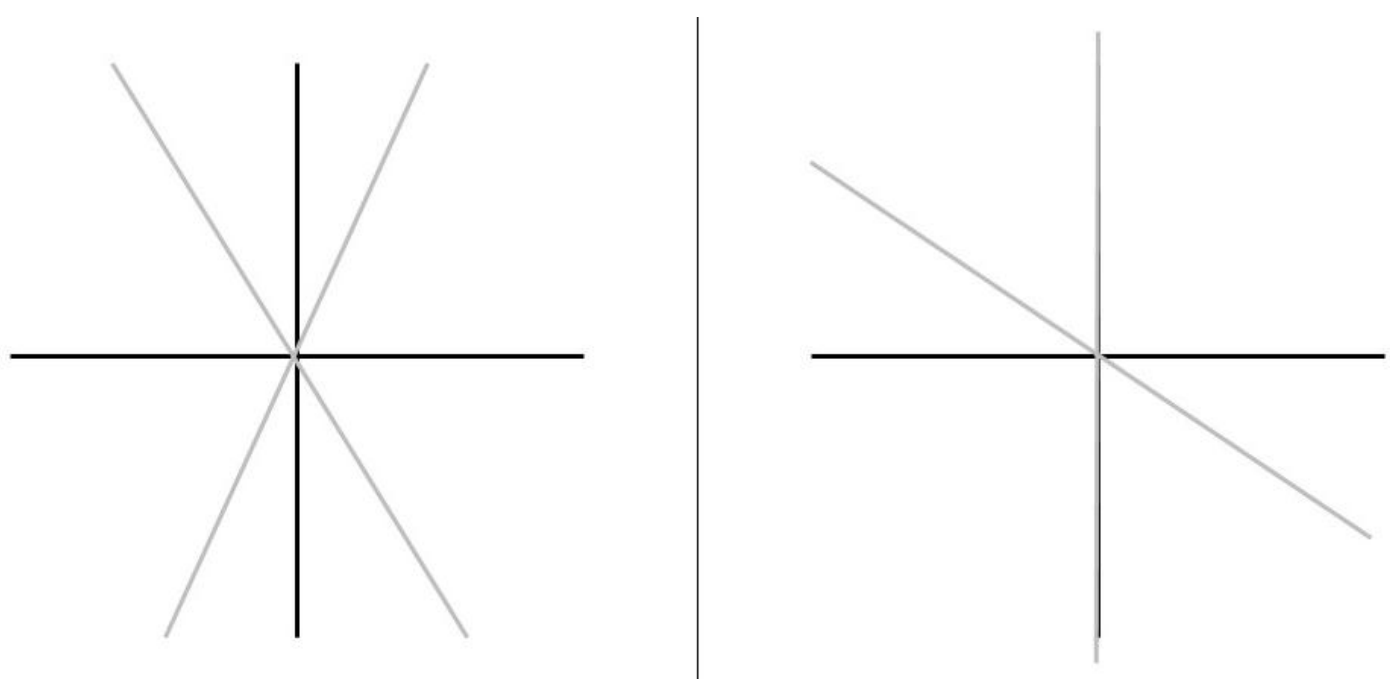

Figure 4. Simplified Flow Diagram of the Orthogonal Procrustes Rotation.

Note. This Figure should be read from left (before Procrustes rotation) to right (after Procrustes rotation). The gray color depicts empirical results, while black depicts theoretical expectations. The relative location of empirical results was unchanged, but was adjusted to be maximally congruent to the theoretical solution.

\subsubsection{Criteria of Evaluation}

To evaluate how well the empirical matrix fits its theoretical counterpart, congruence coefficients are used (Tucker, 1951). In case both matrices would ideally converge, the congruence coefficient would equal 1 . There are two sorts of congruence coefficients: model congruence, informing how well the whole empirical matrix fits the theoretical one, and 
variable congruence, informing how well each variable fits its own hypothesized location. Usually, values of at least .90 (ideally above .95) are considered as high congruence between compared matrices (Barrett, 1986; Lorenzo-Seva, \& ten Berge, 2006).

\section{Example Study}

The main goal of the study is to present the three-step procedure by testing the structure of the CPM measure and examining whether the dimensions of FFNI can be located in CPM, given the fact that many narcissistic constructs were already located within CPM, according to expectations formulated based on NSM and CPM (Rogoza et al., 2019). Thus, we have clear expectations regarding the results (also illustrated on Figure 3), which are: vulnerability should be located near Gamma-Minus (at angular location of $225^{\circ}$ ), antagonism should be located near Alpha-Minus (at angle of $270^{\circ}$ ), and grandiosity should be located near Delta-Minus (at angle $315^{\circ}$ ). Using sine and cosine functions on these angles ${ }^{2}$, they could be transformed to the two-dimensional space in the form of factor loadings. The transformed theoretical matrix is presented in Table 1.

Table 1

Theoretical Matrix Calculated out of the Expected Angular Locations of the Three Facets of Narcissism

\begin{tabular}{lccc}
\hline & Expected angle & Sine & Cosine \\
\hline Vulnerability & 225 & -.71 & -.71 \\
Antagonism & 270 & -1.00 & .00 \\
Grandiosity & 315 & -.71 & .71 \\
\hline
\end{tabular}

To test this hypothesis, we applied the three-step procedure as described above, that is: 1) we verified the circumplex structure of the CPM in SEM (Grassi, Luccio, \& Di Blass,

\footnotetext{
${ }^{2}$ The trigonometric functions are not directly applied to angles. These should first be transformed into radians. Please see the associated tutorial on how to do that.
} 
2010); 2) we tested the possibility to locate FFNI scores in the circumplex space of the CPM using SSM (Zimmerman \& Wright, 2017); and 3) we tested the congruence between empirical locations to theoretical expectations using Procrustes rotation in Orthosim 2.1.a (Barrett, 2013).

\subsection{Tutorials}

This paper has an associated OSF project page, where all of the data and syntaxes are stored, which can be found at: https://osf.io/r95h7. Moreover, for each step of the statistical procedure, we uploaded tutorials illustrating the installation processes, data preparation, using the software, and pedagogical explanation of how to run the analyses on different data.

\section{Method}

\subsection{Participants procedure}

The study was completed online by $N=465$ Polish adults aged from 18 to 70 years old $(M=32.08 ; S D=10.65 ; 28.6 \%$ males $)$. This study was part of a larger data collection effort in which we gathered data on narcissistic personality aiming to develop a new measure. More details about sample and procedure can be found in Rogoza, Cieciuch, \& Strus (2019). Analyses presented in the current paper are novel and overlap between studies with regard to descriptive statistics and reliability estimates.

\subsection{Measures}

\subsubsection{Five Factor Narcissism Inventory}

We used FFNI short form (i.e., comprising 60 items; Sherman et al., 2015). Respondents rate their agreement using a five-point response scale ranging from 1 (Disagree strongly) to 5 (Agree strongly). We calculated three composite scores of grandiosity $(\alpha=.91)$, antagonism ( $\alpha=.89)$, and vulnerability ( $\alpha=.91$; Miller et al., 2016) using modified scoring, 
that is, manipulativeness and thrill seeking were both entered as indicators of grandiosity, not antagonism (Rogoza, Kłosowski, Cieciuch, \& Strus, 2019).

\subsubsection{Circumplex of Personality Metatraits Questionnaire}

The Circumplex of Personality Metatraits Questionnaire comprises 72-items on which respondents rate their agreement using a five-point Likert type response scale ranging from 1 (definitely disagree) to 5 (definitely agree). The reliability estimates were good for all scales: Delta-Plus $(\alpha=.76)$, Alpha-Plus $(\alpha=.70)$, Gamma-Plus $(\alpha=.80)$, Beta-Plus $(\alpha=.81)$, DeltaMinus $(\alpha=.82)$, Alpha-Minus $(\alpha=.82)$, Gamma-Minus $(\alpha=.87)$, and Beta-Minus $(\alpha=.75)$.

\section{Results}

\subsection{Step 1. Verification of the CPM Structure}

The fit indices of SEM model testing the hypothesis about circumplex structure with equal spacing and equal communalities, using three betas in the Fourier correlation ${ }^{3}$, were $\operatorname{good}\left(\chi_{(24)}^{2}=149.60 ; p<.001 ; \mathrm{CFI}=.930 ; \mathrm{GFI}=.937 ; \mathrm{AFGI}=.906 ; \mathrm{RMSEA}=.106\right.$. Thus, the underlying structure of the CPM might be deemed as circumplex ${ }^{4}$.

\subsection{Step 2. Testing the Possibility to Locate an External Variable Within Empirical}

\section{Circumplex}

The projections of the dimensions of narcissism onto the empirical circumplex space of CPM are illustrated on Figure 5, while exact estimates are presented in Table 2. All of the projected variables' correlation profiles were well fitted to the cosine curve, making interpretation of their structural summary meaningful. Neither of the elevation parameters

\footnotetext{
${ }^{3}$ The model with one and two betas represented a worse fit, while the model with four betas in the Fourier correlation was overparametrized and resulted in a lack of convergence.

${ }^{4} \mathrm{We}$ also tested other models, the fit indices of which are as follows: circular $\left(\chi_{(10)}^{2}=71.57 ; p<.001 ; \mathrm{CFI}=\right.$ $.966 ; \mathrm{GFI}=.968 ; \mathrm{AFGI}=.885 ; \mathrm{RMSEA}=.115)$; quasi-circumplex, unequal communalities $\left(\chi_{(17)}^{2}=114.00 ; p<\right.$ $.001 ; \mathrm{CFI}=.946 ; \mathrm{GFI}=.950 ; \mathrm{AFGI}=.894 ; \mathrm{RMSEA}=.111) ;$ quasi-circumplex, unequal spacing $\left(\chi_{(17)}^{2}=\right.$ $108.43 ; p<.001 ; \mathrm{CFI}=.949 ; \mathrm{GFI}=.953 ; \mathrm{AFGI}=.900 ; \mathrm{RMSEA}=.108)$.
} 
were notable, which is not surprising given the fact CPM does not assume the presence of any sort of general factor. All of the amplitude values in turn were notable, meaning that each profile was distinct. The location of antagonism was nearly identical to its hypothesized position, whilst the locations of vulnerability and grandiosity were in high proximity to their hypothesized positions.

Table 2

Structural Summary Profiles of Dimensions of Narcissism Projected on the Circumplex Space of the CPM

\begin{tabular}{lcccc}
\hline Profile & Fit $\left(R^{2}\right)$ & Elevation & Amplitude & Empirical angle \\
\hline Vulnerability & .97 & $.01[-.01, .04]$ & $.43[.36, .50]$ & $216.4[206.6,226.3]$ \\
Antagonism & .97 & $.09[.06, .11]$ & $.49[.43, .55]$ & $267.2[258.8,276.0]$ \\
Grandiosity & .96 & $.11[.08, .14]$ & $.52[.47, .57]$ & $329.9[321.6,338.4]$ \\
\hline
\end{tabular}

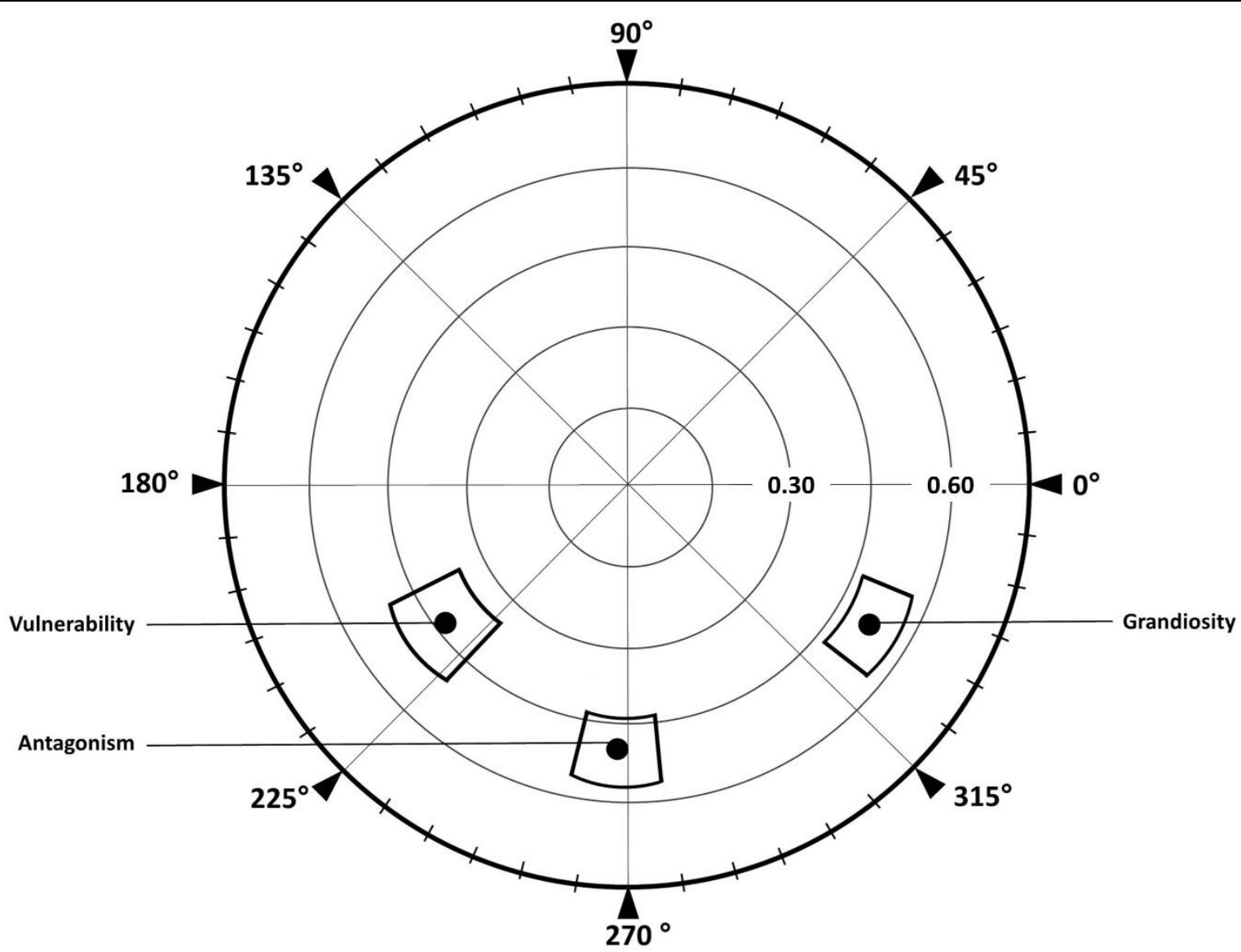


Figure 5. Empirical Projections of the Dimensions of Narcissism on the Empirical

Circumplex Structure of the CPM.

\subsection{Step 3. Testing of the Congruence Between Empirical Locations and Theoretical Predictions Within Circumplex Structure}

As discussed earlier, a theoretical matrix was created on the basis of sines and cosines of theoretical angles of each variable, the estimates of which are reported in Table 2 and a reminder is provided below. Empirical matrices, which will be compared against such a theoretical matrix are presented in Table 3.

Table 3

Empirical Matrix Calculated out of the Estimated Angular Locations of the Three Facets of Narcissism Compared Against Theoretical Matrix

\begin{tabular}{lcccccc}
\hline & & & \multicolumn{2}{c}{ Theoretical matrix } & \multicolumn{2}{c}{ Empirical matrix } \\
\cline { 4 - 7 } & $\begin{array}{c}\text { Theoretical } \\
\text { angle }\end{array}$ & $\begin{array}{c}\text { Empirical angle } \\
{[90 \% \mathrm{CI}]}\end{array}$ & $\begin{array}{c}\text { Expected } \\
\text { sine }\end{array}$ & $\begin{array}{c}\text { Expected } \\
\text { cosine }\end{array}$ & $\begin{array}{c}\text { Sine } \\
{[90 \% \mathrm{CI}]}\end{array}$ & $\begin{array}{c}\text { Cosine } \\
{[90 \% \mathrm{CI}]}\end{array}$ \\
\hline Vulnerability & 225 & 216.4 & -.71 & -.71 & -.59 & -.80 \\
Antagonism & 270 & $\begin{array}{c}{[206.6,226.3]} \\
267.2\end{array}$ & -1.00 & .00 & -1.00 & -.05 \\
& & {$[258.8,276.0]$} & & & {$[-.98,-.99]$} & {$[-.19, .10]$} \\
Grandiosity & 315 & 329.9 & -.71 & .71 & -.50 & .87 \\
& & {$[321.6,338.4]$} & & & {$[-.62,-.37]$} & {$[.78, .93]$} \\
\hline
\end{tabular}

The overall solution congruence $=.98[.98, .99]$ indicated that the location of the NSM, as measured by the FFNI scales, was successfully confirmed. The congruence between each scale to their specific locations were as follows: vulnerability $=.99[.98, .99]$, 
antagonism $=1.00[1.00,1.00]$, and grandiosity $=.97[.97, .97]$. Thus, all of the hypotheses concerning specific scale locations were confirmed as well.

\section{Summary}

Circumplex models are well-known in psychological research (Gurtman \& Pincus, 2003; Wiggins, 1979) and although new measures of their evaluation are constantly being developed (Zimmerman \& Wright, 2017), to date there are no comprehensive procedures for their analysis. In the current paper we propose such a procedure, to facilitate understanding of circumplex models and make their interpretation easier. We propose a three-step evaluation

process consisting of: 1) testing the circumplex structure; 2) testing the possibility to locate an external variable within the empirical circumplex; and 3) testing the level of congruence between empirical locations and a theoretical prediction within the circumplex structure. To date, analyses described in each step have only been used randomly; some of the studies provided only projections (e.g., Grove, Smith, Girard, \& Wright, 2019), while some verified the circumplex structure and provided projections (e.g., Boudreaux, Ozer, Oltmanns, \& Wright, 2018). The results provided by the mentioned studies are valuable, however, under some circumstances (as an example of grandiosity in the current study), the final interpretation is threatened by some dose of subjectivity and further evaluation criteria are needed. We argue that application of the Procrustes rotation makes the procedure comprehensive. The three-step procedure sets a new standard in the evaluation of the circumplex models and the example of assessment of narcissistic personality supported its utility. To facilitate the comprehensiveness of the proposed procedure, in Figure 6 we provide a decision tree, illustrating what to do in the light of obtained results. 


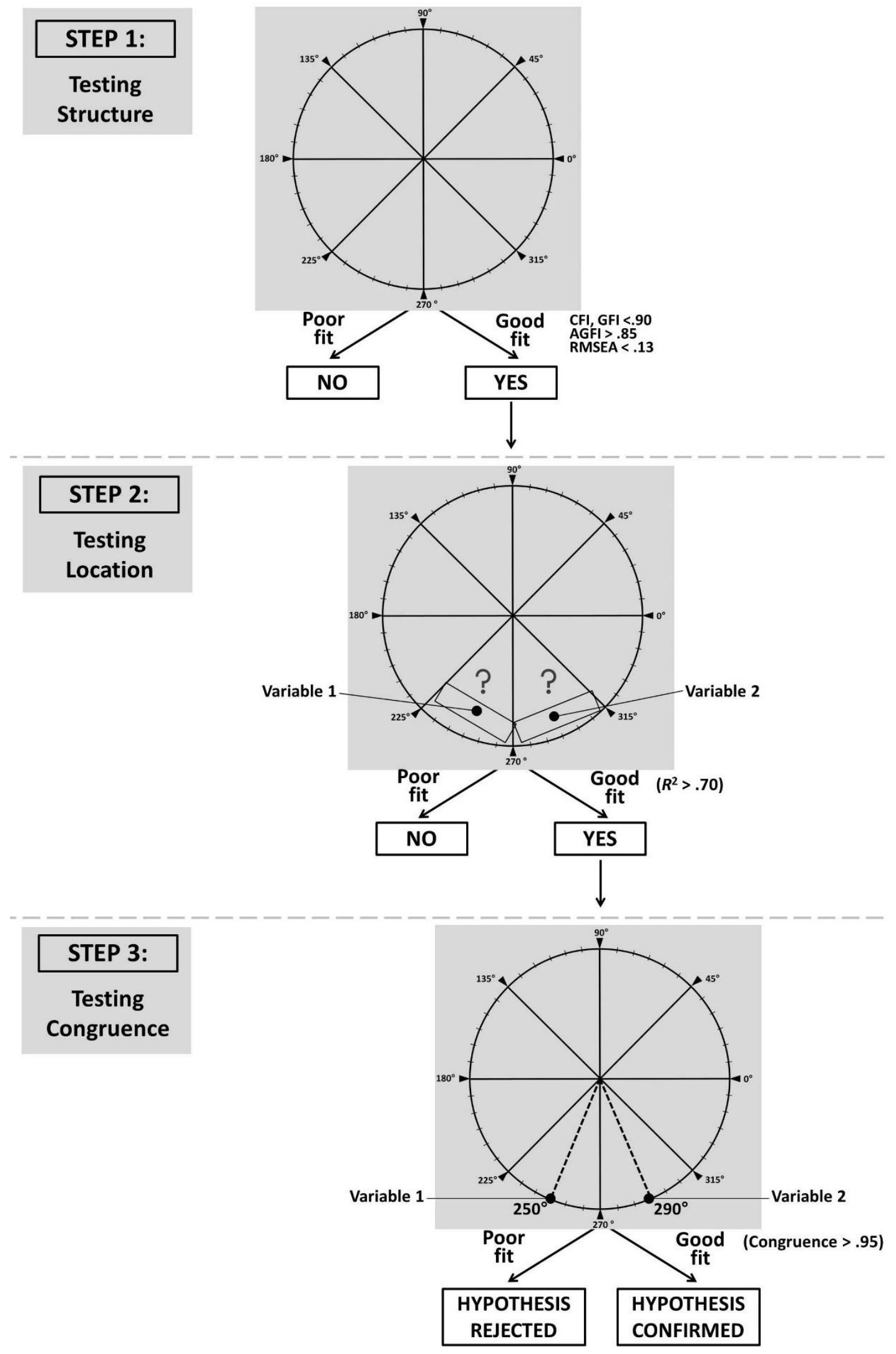

Figure 6. Decision Tree of the Three-Step Procedure for Analysis of Circumplex Models. 
In the example study of how to run the three step procedure, we examined whether narcissism measured by the FFNI (Miller et al., 2016) corresponds to the dimensions of the NSM (Krizan \& Herlache, 2018), which was previously located within the theoretical space of the CPM (Rogoza et al., 2019). To date, there have been theoretical claims suggesting their congruence (Wright \& Edershile, 2018). However, there were limited possibilities regarding how to test that. Following the three-step procedure, we 1) verified the underlying circumplex structure of the CPM, 2) located narcissism measured by FFNI scales in the circumplex structure, and 3) provided evidence that these empirical locations were in high congruence to the theoretical predictions concerning the location of the former within the latter (i.e., the CPM). These results, together with previous results by Rogoza et al. (2019), suggest that FFNI scales could be interpreted as indicators of the NSM dimensions. 


\section{References}

Barrett, P. (1986). Factor comparison: An examination of three methods. Personality and Individual Differences, 7, 327-340. doi:10.1016/0191-8869(86)90008-5

Barrett, P. (2013). Orthosim: Target-Comparison matrix fitting. Retrieved from https://www.pbarrett.net/orthosim/orthosim.html

Boudreaux, M.J., Ozer, D.J., Oltmanns, T.F., \& Wright, A.G.C. (2018). Development and validation of the circumplex scales of interpersonal problems. Psychological Assessment, 30, 594-609. doi:10.1037/pas0000505

Browne, M.W. (1992). Circumplex models for correlation matrices. Psychometrika, 57, 469497. doi: 10.1007/BF02294416

Browne, M.W., MacCallum, R.C., Kim, C.T., Andersen, B.L., \& Glaser, R. (2002). When fit indices and residuals are incompatible. Psychological Methods, 7, 403-421. doi:10.1037/1082-989X.7.4.403

Byrne, B.M. (1994). Structural equation modeling with EQS and EQS/Windows. Thousand Oaks, CA: Sage Publications.

Cieciuch, J., \& Strus, W. (2017) Two-Factor Model of personality. In V. Zeigler-Hill, \& T. Shackelford (Eds.), Encyclopedia of Personality and Individual Differences. Springer International Publishing AG. doi:10.1007/978-3-319-28099-8

Cieciuch, J., \& Topolewska, E. (2017). Circumplex of identity formation modes: A proposal for the integration of identity constructs developed in the Erikson-Marcia tradition. Self and Identity, 16, 37-61. doi:10.1080/15298868.2016.1216008

Crowe, M.L., Lynam, D.R., Campbell, W.K., \& Miller, J.D. (2019). Exploring the structure of narcissism: Towards an integrated solution. Journal of Personality. Advance online publication. doi:10.1111/jopy.12464 
Grassi, M., Luccio, R., \& Di Blas, L. (2010). CircE: An R implementation of Browne's circular stochastic process model. Behavior Research Methods, 42, 55-73. doi:10.3758/BRM.41.1.55

Grove, J.L., Smith, T.W., Girard, J.M., \& Wright, A.G.C. (2019). Narcissistic admiration and rivalry: An interpersonal approach to construct validation. Journal of Personality Disorders, 16, 1-25. doi:10.1521/pedi_2019_33_374

Gurtman, M.B. (1994). The circumplex as a tool for studying normal abnormal personality: A methodological primer. In S. Strack \& M. Lorr (Eds.), Differentiating normal and abnormal personality (pp. 243-263). New York: Springer.

Krizan, Z., \& Herlache, A.D. (2018). The narcissism spectrum model: A synthetic view of narcissistic personality. Personality and Social Psychology Review, 22, 3-31. doi:10.1177/1088868316685018

Lorenzo-Seva, U., ten Berge, J.M.F. (2006). Tucker's congruence coefficient as a meaningful index of factor similarity. Methodology, 2, 57-64. doi:10.1027/1614-1881.2.2.57

McCrae, R.R., \& Costa, P.T. (1997). Personality trait structure as a human universal. American Psychologist, 52, 509-516. doi:10.1037/0003-066X.52.5.509

Miller, J.D., Lynam, D.R., McCain, J.L., Few, L.R., Crego, C., Widiger, T.A., \& Campbell, W.K. (2016). Thinking structurally about narcissism: An examination of the Five-Factor Narcissism Inventory and its components. Journal of Personality Disorders, 29, 1-18. doi:10.1521/pedi_2015_29_177

Paulhus, D.L., \& Williams, K.M. (2002). The Dark Triad of personality: Narcissism, Machiavellianism, and psychopathy. Journal of Research in Personality, 36, 556-563. doi: 10.1016/S0092-6566(02)00505-6

Pincus, A.L., \& Gurtman, M.B. (2003). Interpersonal assessment. In J.S. Wiggins (Ed.), Paradigms of personality assessment (pp. 246-261). New York, N: Guilford. 
Revelle, W., Wilt, J. (2013). The general factor of personality: A general critique. Journal of Research in Personality, 47, 493-504. doi:10.1016/j.jrp.2013.04.012

Rogoza, R., Cieciuch, J., \& Strus, W. (2019). Narcissistic isolation and enmity concept: Disentangling the blue and the dark face of vulnerable narcissism. Manuscript submitted for publication.

Rogoza, R., Cieciuch, J., Strus, W., Baran, T. (2019). Seeking a common framework for research on narcissism: An attempt to integrate the different faces of narcissism within the Circumplex of Personality Metatraits. European Journal of Personality, 33, 437455. doi:10.1002/per.2206

Rogoza, R., Kłosowski, M., Cieciuch, J., \& Strus, W. (2019). Structure of the Polish version of the Five Factor Narcissism Inventory: Insight from the network psychometrics approach. Manuscript submitted for publication.

Rogoza, R., Żemojtel-Piotrowska, M., Kwiatkowska, M.M., \& Kwiatkowska, K. (2018). The bright, the dark, and the blue face of narcissism: The spectrum of narcissism in its relations to the metatraits of personality, self-esteem, and nomological network of shyness, loneliness and empathy. Frontiers in Psychology, 9, 343. doi:10.3389/fpsyg.2018.00343

Russell, J.A. (1980). A Circumplex Model of Affect. Journal of Personality and Social Psychology, 39, 1161-1178. doi:10.1037/h0077714.

Saris, W.E., Satorra, A., \& van der Veld, W.M. (2009). Testing structural equation models or detection of misspecifications? Structural Equation Modeling, 16, 561-582. doi:10.1080/10705510903203433

Schermelleh-Engel, K., Moosbrugger, H., \& Müller, H. (2003). Evaluating the fit of structural equation models: Tests of significance and descriptive goodness-of-fit measures. Methods of Psychological Research Online, 8, 23-74. 
Schwartz, S.H., Cieciuch, J., Vecchione, M., Davidov, E., Fischer, R., Beierlein, C., Ramos, A., Verkasalo, M., Lönnqvist, J.-E., Demirutku, K., Dirilen-Gumus, O., \& Konty, M. (2012). Refining the theory of basic individual values. Journal of Personality and Social Psychology, 103, 663-688. doi:10.1037/a0029393

Sherman, E.D., Miller, J.D., Few, L.R., Campbell, W.K., Widiger, T.A., Crego, C., \& Lynam, D.R. (2015). Development of a short form of the Five-Factor Narcissism Inventory: The FFNI-SF. Psychological Assessment, 27, 1110-1116. doi:10.1037/pas0000100

Strus, W., \& Cieciuch, J. (2017). Towards a synthesis of personality, temperament, motivation, emotion and mental health models within the Circumplex of Personality Metatraits. Journal of Research in Personality, 66, 70-95.

doi:10.1016/j.jrp.2016.12.002

Strus, W., Cieciuch, J., \& Rowiński, T. (2014). The Circumplex of Personality Metatraits: A synthesizing model of personality based on the Big Five. Review of General Psychology, 18, 273-286. doi:10.1037/gpr0000017

Terracciano, A., McCrae, R.R., Hagemann, D., Costa, P.T. (2003). Individual difference variables, affective differentiation, and the structures of affect. Journal of Personality, 71, 669-703. doi:10.1111/1467-6494.7105001

Tracey, T.J.G. (2000). Analysis of circumplex models. In H.E.A. Tinsley \& S.D. Brown (Eds.), Handbook of applied multivariate statistics and mathematical modeling (pp. 641-664). New York: Academic Press. doi:10.1016/B978-012691360-6/50023-9

Tucker, L.R. (1951). A method for synthesis of factor analysis studies (Personnel Research Section Report No. 984). Washington, DC: Department of the Army.

Wiggins, J.S. (1979). A psychological taxonomy of trait-descriptive terms: The interpersonal domain. Journal of Personality and Social Psychology 37, 395-412. 
Wright, A.G.C., \& Edershile, E. (2018). Issues resolved and unresolved in pathological narcissism. Current Opinion in Psychology, 21, 74-79. doi:10.1016/j.copsyc.2017.10.001

Wright, A.G.C., Pincus, A.L., Conroy, D.E., \& Hilsenroth, M.J. (2009). Integrating methods to optimize circumplex description and comparison of groups. Journal of Personality Assessment, 91, 311-322. doi:10.1080/00223890902935696

Zawadzki, B. (2017). The location of personality disorders in the Circumplex of Personality Metatraits. Annals of Psychology, 20, 493-512. doi:10.18290/rpsych.2017.20.2-7en

Zimmerman, J., \& Wright, A.G.C. (2017). Beyond description in interpersonal construct validation: Methodological advances in the circumplex structural summary approach. Assessment, 24, 3-23. doi:10.1177/1073191115621795 